\title{
ON THE DECAY PROCESS OF TURBULENCE AT LARGE REYNOLDS AND PECLET NUMBERS
}

\author{
HIMADRI PAI MAZUMDAR * \\ Dept. of Appl. Math., \\ Twente Univ. of Technology, \\ Enschede, THE NETHERLANDS
}

\begin{abstract}
When fluctuating temperature field is considered to be super imposed on a general field of eddy turbulence, the early period decay phenomena in regard to velocity, temperature and velocity-temperature are guided by three dynamical equations that are obtained here in a straightforward manner. The equations so obtained are simplified for the case of homogeneous turbulence and subsequently for the case of homogeneous and isotropic turbulence.
\end{abstract}

\section{§ 1. Introduction}

The study of the behaviour of a turbulent scalar field e.g. temperature, concentration etc. superimposed on a turbulent velocity field is essential from the view point of turbulent mixing. Due to this, the extension of the theories propounded for the turbulent velocity field to the case of turbulent scalar fields are often desireable. Obukhoff [1] and Corrsin [2] have studied the small scale structure of the fluctuating scalar field with the help of Kolmogoroff's universal equillibrium hypothesis. O'Brien and Francis [3] presented the dynamical consequences of the quasinormality approximation (cf. Millionshtchikov, [4, 5]) which states that the fourth-order moments are related to the second-order moments as in a normal distribution when applied to the behaviour of a scalar field. Following Chandrasekhar's [6] deductive theory of turbulence Jain [7] studied the fluctuating temperature field and compared his results with those of Corrsin [2]. Codreanu [8] obtained the equation for the spectrum of the scalar quantity using the method proposed by Malfliet [9]

* Present adress: Institut für stromungslehre und Gasdynamik. Graz Technical University, Graz, Austria. 
in his study of turbulent velocity field. In most of the investigations both the turbulent velocity field and the turbulent scalar field are assumed statistically homogeneous and isotropic. Kistler, O'Brien and Corrsin [10] obtained an isotropic temperature field superimposed on an isotropic velocity field by introducing a grid with heated bars in their experimental investigation. The present investigation is aimed at deriving the early-period decay equations for general type of turbulence visualised by superimposing a scalar field e.g. the temperature field on an incompressible velocity field. The approach is phenomenological in that we have considered the region where the variations of the mean temperature and the mean velocity may be neglected because the transportation of the thermal energy from place to place is very rapid. Its is assumed throughout the analysis that the observer moves with the mean velocity.

\section{§ 2. Dynamical equations}

Under the assumptions made above the relevant equations pertaining to the point $P(x, t)$ are reducible to the forms

$$
\begin{gathered}
\frac{\partial \theta}{\partial t}+\frac{\partial \theta u_{l}}{\partial x_{l}}=\chi \nabla_{x}^{2} \theta \\
\frac{\partial u_{i}}{\partial t}+\frac{\partial}{\partial x_{l}} u_{i} u_{l}=-\frac{1}{\rho} \frac{\partial p}{\partial x_{i}}+\nu \nabla_{x}^{2} u_{i}
\end{gathered}
$$

where $\theta$ is the temperature fluctuation; $\chi=\alpha / \rho C_{p} ; \alpha$ the coefficient of thermal conductivity; $\rho$ the density; $C_{p}$ the specific heat at constant pressure; $u_{i}$ the fluctuating velocity component; $v$ the kinematic viscosity; $\boldsymbol{x}=\left\{x_{1}, x_{2}, x_{3}\right\}$

$$
\nabla_{x}^{2}=\frac{\partial}{\partial x_{1}^{2}}+\frac{\partial}{\partial x_{2}^{2}}+\frac{\partial}{\partial x_{3}^{2}}
$$

The continuity condition pertaining to the point $P(x, t)$ viz.,

$$
\frac{\partial u_{l}}{\partial x_{l}}=0
$$

has been included in (2.1) and (2.2).

The equation for the temperature fluctuation $\theta^{\prime}$ at the point $P^{\prime}\left(x^{\prime}, t\right)$ similar to that for $\theta$ in (2.1) may be written as

$$
\frac{\partial \theta^{\prime}}{\partial t}+\frac{\partial \theta^{\prime} u_{m}^{\prime}}{\partial x_{m}^{\prime}}=\chi \nabla_{x^{\prime}}^{2} \theta^{\prime}
$$


where

$$
\boldsymbol{x}^{\prime}=\left\{x_{1}^{\prime}, x_{2}^{\prime}, x_{3}^{\prime}\right\} \quad \text { and } \quad \nabla_{x^{\prime}}^{2}=\frac{\partial^{2}}{\partial x_{1}^{\prime 2}}+\frac{\partial^{2}}{\partial x_{2}^{\prime 2}}+\frac{\partial^{2}}{\partial x_{3}^{\prime 2}}
$$

Multiplying (2.1) by $\theta^{\prime}$ and (2.4) by $\theta$, adding the two equations, and on averaging we obtain

$$
\begin{aligned}
\frac{\partial}{\partial t} \overline{\theta \theta^{\prime}}+\frac{\partial}{\partial x_{l}} \overline{\theta u_{l} \theta^{\prime}}+\frac{\partial}{\partial x_{m}^{\prime}} \overline{\theta u_{m}^{\prime} \theta^{\prime}}= & \\
& =\chi\left(\nabla_{x}^{2}+\nabla_{x^{\prime}}^{2}\right) \overline{\theta \theta^{\prime}}
\end{aligned}
$$

In terms of Correlation tensors, (2.5) can be rewritten as

$$
\begin{aligned}
\frac{\partial}{\partial t} F_{\theta, \theta}+\frac{\partial}{\partial x_{l}} F_{\theta l, \theta}+\frac{\partial}{\partial x_{m}^{\prime}} F_{\theta, \theta m} & = \\
& =\chi\left(\nabla_{x}^{2}+\nabla_{x^{\prime}}^{2}\right) F_{\theta, \theta}
\end{aligned}
$$

where

$$
\overline{\theta \theta^{\prime}}=F_{\theta, \theta}\left(\boldsymbol{x}, \boldsymbol{x}^{\prime}, t\right), \quad \overline{\theta u_{l} \theta^{\prime}}=F_{\theta l, \theta}\left(\boldsymbol{x}, \boldsymbol{x}^{\prime}, t\right), \quad \overline{\theta \theta^{\prime} u_{m}^{\prime}}=F_{\theta, \theta m}\left(\boldsymbol{x}, \boldsymbol{x}^{\prime}, t\right)
$$

Using continuity condition for the point $P^{\prime \prime}\left(x^{\prime \prime}, t\right)$, the equation for the velocity fluctuation $u_{k}^{\prime \prime}$ at that point can be written as

$$
\frac{\partial u_{k}^{\prime \prime}}{\partial t}+\frac{\partial}{\partial x_{n}^{\prime \prime}} u_{k}^{\prime \prime} u_{n}^{\prime \prime}=-\frac{\partial}{\partial x_{k}^{\prime \prime}} \omega^{\prime \prime}+v \nabla_{x^{\prime \prime}}^{2} u_{k}^{\prime \prime}
$$

where $\omega^{\prime \prime}=p^{\prime \prime} \mid \rho ; p^{\prime \prime}$ the fluctuating part of pressure at $P^{\prime \prime}\left(x^{\prime \prime}, t\right)$;

$$
\boldsymbol{x}^{\prime \prime}=\left\{x_{1}^{\prime \prime}, x_{2}^{\prime \prime}, x_{3}^{\prime \prime}\right\} ; \quad \nabla_{x^{\prime \prime}}^{2}=\frac{\partial^{2}}{\partial x_{1}^{\prime \prime 2}}+\frac{\partial^{2}}{\partial x_{2}^{\prime \prime 2}}+\frac{\partial^{2}}{\partial x_{3}^{\prime \prime 2}}
$$

Now multiplying (2.1) by $\theta^{\prime} u_{k}^{\prime \prime},(2.4)$ by $\theta u_{k}^{\prime \prime},(2.7)$ by $\theta \theta^{\prime}$ and adding, we obtain, on averaging

$$
\begin{aligned}
& \left.\frac{\partial}{\partial t} \overline{\theta \theta^{\prime} u_{k}^{\prime \prime}}+\frac{\partial}{\partial x_{l}} \overline{\left(\theta u_{l} \theta^{\prime} u_{k}^{\prime \prime}\right.}\right)+\frac{\partial}{\partial x_{m}^{\prime}} \overline{\theta^{\prime} u_{m}^{\prime} \theta u_{k}^{\prime \prime}}+ \\
& +\frac{\partial}{\partial x_{n}^{\prime \prime}} \overline{u_{k}^{\prime \prime} u_{n}^{\prime \prime} \theta \theta^{\prime}}=-\frac{\partial}{\partial x_{k}^{\prime \prime}} \overline{\omega^{\prime \prime} \theta \theta^{\prime}}+\left\{\chi\left(\nabla_{x}^{2}+\nabla_{x^{\prime}}^{2}\right)+v \nabla_{x^{\prime \prime}}^{2}\right\} \overline{\theta \theta^{\prime} u_{k}^{\prime \prime}}
\end{aligned}
$$

Using tensor notations, (2.8) can be rewritten as 


$$
\begin{aligned}
\frac{\partial}{\partial t} F_{\theta, \theta, k}+\frac{\partial}{\partial x_{l}} F_{\theta l, \theta, k}+\frac{\partial}{\partial x_{m}^{\prime}} F_{\theta, \theta m, k}+\frac{\partial}{\partial x_{n}^{\prime \prime}} F_{\theta, \theta, k n}= \\
=-\frac{\partial}{\partial x_{k}^{\prime \prime}} P_{\theta, \theta, 0}+\left\{\chi\left(\nabla_{x}^{2}+\nabla_{x^{\prime}}^{2}\right)+v \nabla_{x^{\prime \prime}}^{2}\right\} F_{\theta, \theta, k}
\end{aligned}
$$

where

$$
\begin{aligned}
& \overline{\theta \theta^{\prime} u_{k}^{\prime \prime}}=F_{\theta, \theta, k}\left(\boldsymbol{x}, \boldsymbol{x}^{\prime}, \boldsymbol{x}^{\prime \prime}, t\right) ; \\
& \overline{\theta u_{l} \theta^{\prime} u_{k}^{\prime \prime}}=F_{\theta l, \theta, k}\left(\boldsymbol{x}, \boldsymbol{x}^{\prime}, \boldsymbol{x}^{\prime \prime}, t\right) ; \overline{\theta^{\prime} u_{m}^{\prime} \theta u_{k}^{\prime \prime}}=F_{\theta, \theta m, k}\left(\boldsymbol{x}, \boldsymbol{x}^{\prime}, \boldsymbol{x}^{\prime \prime}, t\right) ; \\
& \overline{u_{k}^{\prime \prime} u_{n}^{\prime \prime} \theta \theta^{\prime}}=F_{\theta, \theta, k n}\left(\boldsymbol{x}, \boldsymbol{x}^{\prime}, \boldsymbol{x}^{\prime \prime}, t\right) ; \quad \overline{\omega^{\prime \prime} \theta \theta^{\prime}}=P_{\theta, \theta, 0}\left(\boldsymbol{x}, \boldsymbol{x}^{\prime}, \boldsymbol{x}^{\prime \prime}, t\right)
\end{aligned}
$$

Taking $\partial / \partial x_{k}^{\prime \prime}$ of $(2.7)$ and using the continuity condition at $P^{\prime \prime}\left(x^{\prime \prime}, t\right)$ we have

$$
\frac{\partial}{\partial x_{k}^{\prime \prime}} \frac{\partial}{\partial x_{n}^{\prime \prime}} u_{k}^{\prime \prime} u_{n}^{\prime \prime}=-\frac{\partial^{2}}{\partial x_{p}^{\prime \prime} \partial x_{p}^{\prime \prime}} \omega^{\prime \prime}
$$

Multiplaying (2.10) by $\theta \theta^{\prime}$ and on averaging we obtain

$$
\frac{\partial}{\partial x_{k}^{\prime \prime}} \frac{\partial}{\partial x_{n}^{\prime \prime}} \overline{u_{k}^{\prime \prime} u_{n}^{\prime \prime} \theta \theta^{\prime}}=-\frac{\partial^{2}}{\partial x_{p}^{\prime \prime} \partial x_{p}^{\prime \prime}} \overline{\omega^{\prime \prime} \theta \theta^{\prime}}
$$

In tensor notations this relation is

$$
\begin{aligned}
\frac{\partial}{\partial x_{k}^{\prime \prime}} \frac{\partial}{\partial x_{n}^{\prime \prime}} & F_{\theta, \theta, k n}\left(\boldsymbol{x}, \boldsymbol{x}^{\prime}, \boldsymbol{x}^{\prime \prime}, t\right)= \\
& =-\frac{\partial^{2}}{\partial x_{p}^{\prime \prime} \partial x_{p}^{\prime \prime}} P_{\theta, \theta, 0}\left(\boldsymbol{x}, \boldsymbol{x}^{\prime}, \boldsymbol{x}^{\prime \prime}, t\right)
\end{aligned}
$$

Let us introduce the Fourier transforms of two point correlations that appear in (2.5) as

$$
\left.\begin{array}{l}
\psi_{\theta, \theta}\left(K, \boldsymbol{K}^{\prime}, t\right)=\frac{1}{(2 \pi)^{6}} \iint F_{\theta, \theta}\left(\boldsymbol{x}, \boldsymbol{x}^{\prime}, t\right) \exp \left(\mathrm{i}\left[\boldsymbol{K} \cdot \boldsymbol{x}+\boldsymbol{K}^{\prime} \cdot \boldsymbol{x}^{\prime}\right]\right) \mathrm{d} \boldsymbol{x} \mathrm{d} \boldsymbol{x}^{\prime}, \\
\Psi_{\theta l, \theta}\left(\boldsymbol{K}, \boldsymbol{K}^{\prime}, t\right)=\frac{\mathrm{i}}{(2 \pi)^{6}} \iint F_{\theta l, \theta}\left(\boldsymbol{x}, \boldsymbol{x}^{\prime}, t\right) \exp \left(\mathrm{i}\left(\boldsymbol{K} \cdot \boldsymbol{x}+\boldsymbol{K}^{\prime} \boldsymbol{x}^{\prime}\right]\right) \mathrm{d} \boldsymbol{x} \mathrm{d} \boldsymbol{x}^{\prime}, \\
\psi_{\theta, \theta m}\left(\boldsymbol{K}, \boldsymbol{K}^{\prime}, t\right)=\frac{\mathrm{i}}{(2 \pi)^{6}} \iint F_{\theta, \theta m}\left(\boldsymbol{x}, \boldsymbol{x}^{\prime}, t\right) \exp \left(\mathrm{i}\left[\boldsymbol{K} \cdot \boldsymbol{x}+\boldsymbol{K}^{\prime} \cdot \boldsymbol{x}^{\prime}\right]\right) \mathrm{d} \boldsymbol{x} \mathrm{d} \boldsymbol{x}^{\prime},
\end{array}\right\}
$$


Accordingly, the Fourier inverses of the above relations are

$$
\left.\begin{array}{l}
F_{\theta, \theta}\left(\boldsymbol{x}, \boldsymbol{x}^{\prime}, t\right)=\iint \psi_{\theta, \theta}\left(\boldsymbol{K}, \boldsymbol{K}^{\prime}, t\right) \exp \left(-\mathrm{i}\left[\boldsymbol{K} \cdot \boldsymbol{x}+\boldsymbol{K}^{\prime} \cdot \boldsymbol{x}^{\prime}\right]\right) \mathrm{d} \boldsymbol{K} \mathrm{d} \boldsymbol{K}^{\prime}, \\
F_{\theta l, \theta}\left(\boldsymbol{x}, \boldsymbol{x}^{\prime}, t\right)=-\mathrm{i} \iint \psi_{\theta l, \theta}\left(\boldsymbol{K}, \boldsymbol{K}^{\prime}, t\right) \exp \left(-\mathrm{i}\left[\boldsymbol{K} \cdot \boldsymbol{x}+\boldsymbol{K}^{\prime} \cdot \boldsymbol{x}^{\prime}\right]\right) \mathrm{d} \boldsymbol{K} \mathrm{d} \boldsymbol{K}^{\prime}, \\
F_{\theta, \theta m}\left(\boldsymbol{x}, \boldsymbol{x}^{\prime}, t\right)=-\mathrm{i} \iint \psi_{\theta, \theta m}\left(\boldsymbol{K}, \boldsymbol{K}^{\prime}, t\right) \exp \left(-\mathrm{i}\left[\boldsymbol{K} \cdot \boldsymbol{x}+\boldsymbol{K}^{\prime} \cdot \boldsymbol{x}^{\prime}\right]\right) \mathrm{d} \boldsymbol{K} \mathrm{d} \boldsymbol{K}^{\prime},
\end{array}\right\}
$$

It is to be noted that the integrations in (2.12) are performed over the whole of $\boldsymbol{x}, \boldsymbol{x}^{\prime}$-spaces and their respective volume elements are

$$
\mathrm{d} \boldsymbol{x}=\mathrm{d} x_{1} \mathrm{~d} x_{2} \mathrm{~d} x_{3} \text { and } \mathrm{d} \boldsymbol{x}^{\prime}=\mathrm{d} x_{1}^{\prime} \mathrm{d} x_{2}^{\prime} \mathrm{d} x_{3}^{\prime}
$$

Obviously the integration in (2.13) are performed over the whole of $K, K^{\prime}$-spaces and their respective volume elements are

$$
\mathrm{d} \boldsymbol{K}=\mathrm{d} K_{1} \mathrm{~d} K_{2} \mathrm{~d} K_{3} \quad \text { and } \mathrm{d} \boldsymbol{K}^{\prime}=\mathrm{d} K_{1}^{\prime} \mathrm{d} K_{2}^{\prime} \mathrm{d} K_{3}^{\prime}
$$

Similarly, the Fourier transforms of three point correlation tensors that appear in (2.9) may be introduced as

$$
\begin{aligned}
\psi_{\theta, \theta, k}\left(\boldsymbol{K}, \boldsymbol{K}^{\prime}, \boldsymbol{K}^{\prime \prime}, t\right)= & \frac{\mathrm{i}}{(2 \pi)^{9}} \iiint F_{\theta, \theta, k}\left(\boldsymbol{x}, \boldsymbol{x}^{\prime}, \boldsymbol{x}^{\prime \prime}, \mathrm{t}\right) \cdot \\
& \cdot \exp \left(\mathrm{i}\left[\boldsymbol{K} \cdot \boldsymbol{x}+\boldsymbol{K}^{\prime} \cdot \boldsymbol{x}^{\prime}+\boldsymbol{K}^{\prime \prime} \cdot \boldsymbol{x}^{\prime \prime}\right]\right) \mathrm{d} \boldsymbol{x} \mathrm{d} \boldsymbol{x}^{\prime} \mathrm{d} \boldsymbol{x}^{\prime \prime} \\
\psi_{\theta l, \theta, k}\left(\boldsymbol{K}, \boldsymbol{K}^{\prime}, \boldsymbol{K}^{\prime \prime}, t\right)= & \frac{1}{(2 \pi)^{9}} \iiint F_{\theta l, \boldsymbol{\theta}, k}\left(\boldsymbol{x}, \boldsymbol{x}^{\prime}, \boldsymbol{x}^{\prime \prime}, t\right) \cdot \\
& \cdot \exp \left(\mathrm{i}\left[\boldsymbol{K} \cdot \boldsymbol{x}+\boldsymbol{K}^{\prime} \cdot \boldsymbol{x}^{\prime}+\boldsymbol{K}^{\prime \prime} \cdot \boldsymbol{x}^{\prime \prime}\right]\right) \mathrm{d} \boldsymbol{x} \mathrm{d} \boldsymbol{x}^{\prime} \mathrm{d} \boldsymbol{x}^{\prime \prime}
\end{aligned}
$$

and two other similar relations for $\psi_{\theta, \theta m, k}$ and $\psi_{\theta, \theta, k n}$.

Following the same procedure we further introduce

$$
\begin{aligned}
\pi_{\theta, \theta, 0}\left(K, \boldsymbol{K}^{\prime}, \boldsymbol{K}^{\prime \prime}, t\right)= & \frac{1}{(2 \pi)^{9}} \iiint P_{\theta, \theta, 0}\left(\boldsymbol{x}, \boldsymbol{x}^{\prime}, \boldsymbol{x}^{\prime \prime}, t\right) \\
& \cdot \exp \left(\mathrm{i}\left[\boldsymbol{K} \cdot \boldsymbol{x}+\boldsymbol{K}^{\prime} \cdot \boldsymbol{x}^{\prime}+\boldsymbol{K}^{\prime \prime} \cdot \boldsymbol{x}^{\prime \prime}\right]\right) \mathrm{d} \boldsymbol{x} \mathrm{d} \boldsymbol{x}^{\prime} \mathrm{d} \boldsymbol{x}^{\prime \prime}
\end{aligned}
$$

The inverse Fourier transforms of the above relations are

$$
\begin{aligned}
F_{\theta, \theta, k}\left(\boldsymbol{x}, \boldsymbol{x}^{\prime}, \boldsymbol{x}^{\prime \prime}, t\right) & =-\mathrm{i} \iiint \psi_{\theta, \theta, k}\left(\boldsymbol{K}, \boldsymbol{K}^{\prime}, \boldsymbol{K}^{\prime \prime}, t\right) \\
\cdot & \exp \left(-\mathrm{i}\left[\boldsymbol{K} \cdot \boldsymbol{x}+\boldsymbol{K}^{\prime} \cdot \boldsymbol{x}^{\prime}+\boldsymbol{K}^{\prime \prime} \cdot \boldsymbol{x}^{\prime \prime}\right]\right) \mathrm{d} \boldsymbol{K} \mathrm{d} \boldsymbol{K}^{\prime} \mathrm{d} \boldsymbol{K}^{\prime \prime}
\end{aligned}
$$




$$
\begin{aligned}
& F_{\theta l, \theta, k}\left(\boldsymbol{x}, \boldsymbol{x}^{\prime}, \boldsymbol{x}^{\prime \prime}, t\right)=\iiint \psi_{\theta l, \theta, k}\left(\boldsymbol{K}, \boldsymbol{K}^{\prime}, \boldsymbol{K}^{\prime \prime}, t\right) \cdot \\
& \cdot \exp \left(-\mathrm{i}\left[\boldsymbol{K} \cdot \boldsymbol{x}+\boldsymbol{K}^{\prime} \cdot \boldsymbol{x}^{\prime}+\boldsymbol{K}^{\prime \prime} \cdot \boldsymbol{x}^{\prime \prime}\right]\right) \mathrm{d} \boldsymbol{K} \mathrm{d} \boldsymbol{K}^{\prime} \mathrm{d} \boldsymbol{K}^{\prime \prime}
\end{aligned}
$$

with two other similar expressions for $F_{\theta, \theta m, k}$ and $F_{\theta, \theta, k n}$ and the relation

$$
\begin{aligned}
P_{\theta, \theta, 0}\left(x, x^{\prime}, x^{\prime \prime}, t\right) & =\iiint \pi_{\theta, \theta, 0}\left(K, K^{\prime}, K^{\prime \prime}, t\right) \cdot \\
\cdot & \exp \left(-\mathrm{i}\left[\boldsymbol{K} \cdot \boldsymbol{x}+\boldsymbol{K}^{\prime} \cdot \boldsymbol{x}^{\prime}+\boldsymbol{K}^{\prime \prime} \cdot \boldsymbol{x}^{\prime \prime}\right]\right) \cdot \mathrm{d} \boldsymbol{K} \mathrm{d} \boldsymbol{K}^{\prime} \mathrm{d} \boldsymbol{K}^{\prime \prime}
\end{aligned}
$$

Using the relations given in (2.13), (2.6) can be written in the transformed space as

$$
\begin{array}{r}
\frac{\partial}{\partial t} \psi_{\theta, \theta}\left(\boldsymbol{K}, \boldsymbol{K}^{\prime}, t\right)=K_{l} \psi_{\theta l, \theta}\left(\boldsymbol{K}, \boldsymbol{K}^{\prime}, t\right)+\boldsymbol{K}_{m}^{\prime} \psi_{\theta, \theta m}\left(\boldsymbol{K}, \boldsymbol{K}^{\prime}, t\right)- \\
-\chi\left(\boldsymbol{K}^{2}+\boldsymbol{K}^{\prime 2}\right) \psi_{\theta, \theta}\left(\boldsymbol{K}, \boldsymbol{K}^{\prime}, t\right)
\end{array}
$$

Similarly, taking help of the relations (2.17) to (2.19), the equations (2.9) and (2.11) can be rewritten in the wave number space as

$$
\begin{aligned}
& \frac{\partial}{\partial t} \psi_{\theta, \theta, k}\left(\boldsymbol{K}, \boldsymbol{K}^{\prime}, \boldsymbol{K}^{\prime \prime}, t\right)+K_{l} \psi_{\theta l, \theta, k}\left(\boldsymbol{K}, \boldsymbol{K}^{\prime}, \boldsymbol{K}^{\prime \prime}, t\right)+ \\
& +\boldsymbol{K}_{m}^{\prime} \psi_{\theta, \theta m, k}\left(\boldsymbol{K}, \boldsymbol{K}^{\prime}, \boldsymbol{K}^{\prime \prime}, t\right)+K_{n}^{\prime \prime} \psi_{\theta, \theta, k n}\left(\boldsymbol{K}, \boldsymbol{K}^{\prime}, \boldsymbol{K}^{\prime \prime}, t\right)+ \\
& +\boldsymbol{K}_{k}^{\prime \prime} \pi_{\theta, \theta, 0}\left(\boldsymbol{K}, \boldsymbol{K}^{\prime}, \boldsymbol{K}^{\prime \prime}, t\right)=-\left\{\chi\left(K^{2}+\boldsymbol{K}^{\prime 2}\right)+v K^{\prime \prime 2}\right\} \\
& \cdot \psi_{\theta, \theta, k}\left(\boldsymbol{K}, \boldsymbol{K}^{\prime}, \boldsymbol{K}^{\prime \prime}, t\right)
\end{aligned}
$$

and

$$
\frac{K_{k}^{\prime \prime} K_{r}^{\prime \prime} K_{n}^{\prime \prime}}{K^{\prime 2}} \psi_{\theta, \theta, r n}\left(K, K^{\prime}, K^{\prime \prime}, t\right)=-K_{k}^{\prime \prime} \pi_{\theta, \theta, 0}\left(K, K^{\prime}, K^{\prime \prime}, t\right)
$$

Substituting (2.22) in (2.21) we obtain

$$
\begin{aligned}
& \frac{\partial}{\partial t} \psi_{\theta, \theta, k}\left(\boldsymbol{K}, \boldsymbol{K}^{\prime}, \boldsymbol{K}^{\prime \prime}, t\right)+\boldsymbol{K}_{l} \psi_{\theta l, \theta, k}\left(\boldsymbol{K}, \boldsymbol{K}^{\prime}, \boldsymbol{K}^{\prime \prime}, t\right)+ \\
& +K_{m}^{\prime} \psi_{\theta, \theta m, k}\left(\boldsymbol{K}, \boldsymbol{K}^{\prime}, \boldsymbol{K}^{\prime \prime}, t\right)+K_{n}^{\prime \prime} \psi_{\theta, \theta, k n}\left(\boldsymbol{K}, \boldsymbol{K}^{\prime}, \boldsymbol{K}^{\prime \prime}, t\right)- \\
& -\frac{K_{k}^{\prime \prime} K_{r}^{\prime \prime} K_{n}^{\prime \prime}}{K^{\prime \prime 2}} \psi_{\theta, \theta, r n}\left(\boldsymbol{K}, \boldsymbol{K}^{\prime}, \boldsymbol{K}^{\prime \prime}, t\right)=-\left\{\chi\left(K^{2}+K^{\prime 2}\right)+v K^{\prime \prime 2}\right\} \\
& \cdot \psi_{\theta, \theta, k}\left(\boldsymbol{K}, \boldsymbol{K}^{\prime}, \boldsymbol{K}^{\prime \prime}, t\right)
\end{aligned}
$$

Let us consider the case when both Reynolds and Peclet numbers are 
very large, so that the molecular effects are negligible (cf. Panchev, [11]). Under such circumstances we may put $\chi=v=0$ and the equations (2.20) and (2.23) are reduced respectively to the forms

$$
\frac{\partial}{\partial t} \psi_{\theta, \theta}\left(\boldsymbol{K}, \boldsymbol{K}^{\prime}, t\right)=K_{l} \psi_{\theta l, \theta}\left(\boldsymbol{K}, \boldsymbol{K}^{\prime}, t\right)+K_{m}^{\prime} \psi_{\theta, \theta m}\left(\boldsymbol{K}, \boldsymbol{K}^{\prime}, t\right)
$$

and

$$
\begin{aligned}
\frac{\partial}{\partial t} \psi_{\theta, \theta, k}\left(\boldsymbol{K}, \boldsymbol{K}^{\prime}, \boldsymbol{K}^{\prime \prime}, t\right)=-\boldsymbol{K}_{l} \psi_{\theta l, \theta, k}\left(\boldsymbol{K}, \boldsymbol{K}^{\prime}, \boldsymbol{K}^{\prime \prime}, t\right)- \\
\quad-\boldsymbol{K}_{m}^{\prime} \psi_{\theta, \theta m, k}\left(\boldsymbol{K}, \boldsymbol{K}^{\prime}, \boldsymbol{K}^{\prime \prime}, t\right)-K_{n}^{\prime \prime} \psi_{\theta, \theta, k n}\left(\boldsymbol{K}, \boldsymbol{K}^{\prime}, \boldsymbol{K}^{\prime \prime}, t\right)+ \\
\quad+\frac{\boldsymbol{K}_{k}^{\prime \prime} K_{r}^{\prime \prime} K_{n}^{\prime \prime}}{K^{\prime \prime 2}} \psi_{\theta, \theta, r n}\left(\boldsymbol{K}, \boldsymbol{K}^{\prime}, \boldsymbol{K}^{\prime \prime}, t\right)
\end{aligned}
$$

We now consider a few important relations that can be easily proved following Ghosh [12] for use in the subsequent analysis.

The triple correlation $\overline{\theta \theta^{\prime} u_{k}^{\prime \prime}}=F_{\theta, \theta, k}\left(\boldsymbol{x}, \boldsymbol{x}^{\prime}, \boldsymbol{x}^{\prime \prime}, t\right)$ formed of the two temperature fluctuations pertaining to the points $P, P^{\prime}$ and one velocity component pertaining to the point $P^{\prime \prime}$, and its Fourier transform $\psi_{\theta, \theta, k}\left(K, K^{\prime}, K^{\prime \prime}, t\right)$ is being reconsidered as follows.

When the point $P^{\prime \prime}$ concides with the point $P$ or in the alternative $P^{\prime \prime}$ coincides with the point $P^{\prime}$, we respectively obtain the relations,

and

$$
\int \psi_{\theta, \theta, k}\left(\boldsymbol{K}-\boldsymbol{K}^{\prime \prime}, \boldsymbol{K}^{\prime}, \boldsymbol{K}^{\prime \prime}, t\right) \mathrm{d} \boldsymbol{K}^{\prime \prime}=\psi_{\theta k, \theta}\left(\boldsymbol{K}, \boldsymbol{K}^{\prime}, t\right)
$$

$$
\int \psi_{\theta, \theta, k}\left(\boldsymbol{K}, \boldsymbol{K}^{\prime}-\boldsymbol{K}^{\prime \prime}, \boldsymbol{K}^{\prime \prime}, t\right) \mathrm{d} \boldsymbol{K}^{\prime \prime}=\psi_{\theta, \theta k}\left(\boldsymbol{K}, \boldsymbol{K}^{\prime}, t\right)
$$

Let us consider the velocity component $u_{l}^{\prime \prime \prime}$ at a fourth point $P^{\prime \prime \prime}\left(\boldsymbol{x}^{\prime \prime \prime}, t\right)$ and form the quadrulple correlation $\overline{\theta \theta^{\prime} u_{k}^{\prime \prime} u_{l}^{\prime \prime \prime}}=F_{\theta, \theta, k, l}\left(x, x^{\prime}, x^{\prime \prime}, x^{\prime \prime \prime}, t\right)$. The Fourier transform of $F_{\theta, \theta, k, l}\left(x, x^{\prime}, x^{\prime \prime}, x^{\prime \prime \prime}, t\right)$ in wave number is as usual denoted by $\psi_{\theta, \theta, k, l}\left(\boldsymbol{K}, \boldsymbol{K}^{\prime}, \boldsymbol{K}^{\prime \prime}, \boldsymbol{K}^{\prime \prime \prime}, t\right)$.

The quasi-normality hypothesis as required may be expressed in wave number space by the relation

$$
\begin{aligned}
& \psi_{\theta, \theta, k, l}\left(\boldsymbol{K}, \boldsymbol{K}^{\prime}, \boldsymbol{K}^{\prime \prime}, \boldsymbol{K}^{\prime \prime \prime}, t\right)=\psi_{\theta, \theta}\left(\boldsymbol{K}, \boldsymbol{K}^{\prime}, t\right) \psi_{k, l}\left(\boldsymbol{K}^{\prime \prime}, \boldsymbol{K}^{\prime \prime \prime}, t\right)+ \\
& \quad+\psi_{\theta, k}\left(\boldsymbol{K}, \boldsymbol{K}^{\prime \prime}, t\right) \psi_{\theta, l}\left(\boldsymbol{K}^{\prime}, \boldsymbol{K}^{\prime \prime \prime}, t\right)+\psi_{\theta, l}\left(\boldsymbol{K}, \boldsymbol{K}^{\prime \prime \prime}, t\right) \psi_{\theta, k}\left(\boldsymbol{K}^{\prime}, \boldsymbol{K}^{\prime \prime}, t\right)
\end{aligned}
$$

Equation (2.27) may be rewritten when $P^{\prime \prime \prime}$ coincides with $P$ as 


$$
\begin{aligned}
& \psi_{\theta l, \theta, k}\left(\boldsymbol{K}, \boldsymbol{K}^{\prime}, \boldsymbol{K}^{\prime \prime}, t\right)=\int \psi_{\theta, \theta}\left(\boldsymbol{K}-\boldsymbol{K}^{\prime \prime \prime}, \boldsymbol{K}^{\prime}, t\right) \psi_{k, l}\left(\boldsymbol{K}^{\prime \prime}, \boldsymbol{K}^{\prime \prime \prime}, t\right) \mathrm{d} \boldsymbol{K}^{\prime \prime \prime}+ \\
& +\int \psi_{\theta, k}\left(\boldsymbol{K}-\boldsymbol{K}^{\prime \prime \prime}, \boldsymbol{K}^{\prime \prime}, t\right) \psi_{\theta, l}\left(\boldsymbol{K}^{\prime}, \boldsymbol{K}^{\prime \prime \prime}, t\right) \mathrm{d} \boldsymbol{K}^{\prime \prime \prime}+\psi_{\theta, k}\left(\boldsymbol{K}^{\prime}, \boldsymbol{K}^{\prime \prime}, t\right) \\
& \quad \psi_{\theta l}(\boldsymbol{K}, t)
\end{aligned}
$$

Similar relations for $\psi_{\theta, \theta, k l}\left(K, K^{\prime}, K^{\prime \prime}, t\right)$, when $P^{\prime \prime \prime}$ coincides with $P^{\prime \prime}$ and for $\psi_{\theta, \theta l, k}\left(K, K^{\prime}, K^{\prime \prime}, t\right)$ when $P^{\prime \prime \prime}$ coincides with $P^{\prime}$ may be easily obtained. Asymptotic form of (2.28) when in addition to the merging of $P^{\prime \prime \prime}$ with $P, P^{\prime \prime}$ also coinsides with $P^{\prime}$ may be written as

$$
\begin{aligned}
& \psi_{\theta l, \theta k}\left(\boldsymbol{K}, \boldsymbol{K}^{\prime}, t\right)=\iint\left[\psi_{\theta, \theta}\left(\boldsymbol{K}-\boldsymbol{K}^{\prime \prime \prime}, \boldsymbol{K}^{\prime}-\boldsymbol{K}^{\prime \prime}, t\right) \psi_{k, l}\left(\boldsymbol{K}^{\prime \prime}, \boldsymbol{K}^{\prime \prime \prime}, t\right)+\right. \\
& \left.+\psi_{\theta, k}\left(\boldsymbol{K}-\boldsymbol{K}^{\prime \prime \prime}, \boldsymbol{K}^{\prime \prime}, t\right) \psi_{\theta, l}\left(\boldsymbol{K}^{\prime}-\boldsymbol{K}^{\prime \prime}, \boldsymbol{K}^{\prime \prime \prime}, t\right)\right] \mathrm{d} \boldsymbol{K}^{\prime \prime} \mathrm{d} \boldsymbol{K}^{\prime \prime \prime}+ \\
& +\psi_{\theta l}(\boldsymbol{K}, t) \psi_{\theta k}\left(\boldsymbol{K}^{\prime}, t\right)
\end{aligned}
$$

Employing equation (2.25) and the relations (2.26) to (2.29); we derive two expressions for

$$
\frac{\partial}{\partial t} \psi_{\theta l, \theta}\left(\boldsymbol{K}, \boldsymbol{K}^{\prime}, t\right) \text { and } \frac{\partial}{\partial t} \psi_{\theta, \theta m}\left(\boldsymbol{K}, \boldsymbol{K}^{\prime}, t\right)
$$

By taking $\partial / \partial t$ of (2.24) we substitute the expressions for

$$
\frac{\partial}{\partial t} \psi_{\theta l, \theta}\left(\boldsymbol{K}, \boldsymbol{K}^{\prime}, t\right) \quad \text { and } \quad \frac{\partial}{\partial t} \psi_{\theta, \theta m}\left(\boldsymbol{K}, \boldsymbol{K}^{\prime}, t\right)
$$

to obtain an equation guiding the early period decay process as

$$
\frac{\partial^{2}}{\partial t^{2}} \psi_{\theta, \theta}\left(\boldsymbol{K}, \boldsymbol{K}^{\prime}, t\right)=A_{1}\left(\boldsymbol{K}, \boldsymbol{K}^{\prime}, t\right)+A_{2}\left(\boldsymbol{K}, \boldsymbol{K}^{\prime}, t\right)
$$

where

$$
\begin{aligned}
& A_{1}\left(\boldsymbol{K}, \boldsymbol{K}^{\prime}, t\right)=-2 K_{l} K_{m}^{\prime} \iint\left[\psi_{\theta, \theta}\left(\boldsymbol{K}-\boldsymbol{K}^{\prime \prime \prime}, \boldsymbol{K}^{\prime}-\boldsymbol{K}^{\prime \prime}, t\right) \psi_{m, l}\left(\boldsymbol{K}^{\prime \prime}, \boldsymbol{K}^{\prime \prime \prime}, t\right)+\right. \\
& \left.+\psi_{\theta, m}\left(\boldsymbol{K}-\boldsymbol{K}^{\prime \prime \prime}, \boldsymbol{K}^{\prime \prime}, t\right) \psi_{\theta, l}\left(\boldsymbol{K}^{\prime}-\boldsymbol{K}^{\prime \prime}, \boldsymbol{K}^{\prime \prime}, t\right)\right] \mathrm{d} \boldsymbol{K}^{\prime \prime \prime} \mathrm{d} \boldsymbol{K}^{\prime \prime}- \\
& \quad-\int K_{l}\left(K_{t}-\boldsymbol{K}_{t}^{\prime \prime}\right)\left[\int \left\{\psi_{\theta, \theta}\left(\boldsymbol{K}-\boldsymbol{K}^{\prime \prime}-\boldsymbol{K}^{\prime \prime \prime}, \boldsymbol{K}^{\prime}, t\right) \psi_{l, t}\left(\boldsymbol{K}^{\prime \prime}, \boldsymbol{K}_{, \prime \prime} t\right)+\right.\right. \\
& \left.\left.+\psi_{\theta, l}\left(\boldsymbol{K}-\boldsymbol{K}^{\prime \prime}-\boldsymbol{K}^{\prime \prime \prime}, \boldsymbol{K}^{\prime \prime}, t\right) \psi_{\theta, t}\left(\boldsymbol{K}^{\prime}, \boldsymbol{K}^{\prime \prime \prime}, t\right)\right\} \mathrm{d} \boldsymbol{K}^{\prime \prime \prime}\right] \mathrm{d} \boldsymbol{K}^{\prime \prime}-
\end{aligned}
$$




$$
\begin{aligned}
& -\int K_{l} K_{n}^{\prime \prime}\left[\partial_{l r}-\frac{K_{l}^{\prime \prime} K_{r}^{\prime \prime}}{K^{\prime \prime 2}}\right]\left[\int \left\{\psi_{\theta, r}\left(\boldsymbol{K}-\boldsymbol{K}^{\prime \prime}, \boldsymbol{K}^{\prime \prime}-\boldsymbol{K}^{\prime \prime \prime}, t\right) \psi_{\theta, n}\left(\boldsymbol{K}^{\prime}, \boldsymbol{K}^{\prime \prime \prime}, t\right)+\right.\right. \\
& \left.\left.+\psi_{\theta, n}\left(\boldsymbol{K}-\boldsymbol{K}^{\prime \prime}, \boldsymbol{K}^{\prime \prime \prime}, t\right) \psi_{\theta, \boldsymbol{r}}\left(\boldsymbol{K}^{\prime}, \boldsymbol{K}^{\prime \prime}-\boldsymbol{K}^{\prime \prime \prime}, t\right)\right\} \mathrm{d} \boldsymbol{K}^{\prime \prime \prime}\right] \mathrm{d} \boldsymbol{K}^{\prime \prime}- \\
& -\int K_{m}^{\prime}\left(\boldsymbol{K}_{s}^{\prime}-K_{s}^{\prime \prime}\right)\left[\int \left\{\psi_{\theta, \theta}\left(\boldsymbol{K}, \boldsymbol{K}^{\prime}-\boldsymbol{K}^{\prime \prime}-\boldsymbol{K}^{\prime \prime \prime}, t\right) \psi_{m, s}\left(\boldsymbol{K}^{\prime \prime}, \boldsymbol{K}^{\prime \prime \prime}, t\right)+\right.\right. \\
& \left.\left.+\psi_{\theta, s}\left(\boldsymbol{K}, \boldsymbol{K}^{\prime \prime \prime}, t\right) \psi_{\theta, m}\left(\boldsymbol{K}^{\prime}-\boldsymbol{K}^{\prime \prime}-\boldsymbol{K}^{\prime \prime \prime}, \boldsymbol{K}^{\prime \prime}, t\right)\right\} \mathrm{d} \boldsymbol{K}^{\prime \prime \prime}\right] \mathrm{d} \boldsymbol{K}^{\prime \prime}- \\
& -\int K_{m}^{\prime} K_{s}^{\prime \prime}\left[\delta_{m r}-\frac{K_{m}^{\prime \prime} K_{r}^{\prime \prime}}{K^{\prime \prime 2}}\right]\left[\int \left\{\psi_{\theta, \boldsymbol{r}}\left(\boldsymbol{K}_{,} \boldsymbol{K}^{\prime \prime}-\boldsymbol{K}^{\prime \prime \prime}, t\right) \psi_{\theta, s}\right.\right. \\
& +\left(\boldsymbol{K}^{\prime}-\boldsymbol{K}^{\prime \prime}, \boldsymbol{K}^{\prime \prime \prime}, t\right)+ \\
& \left.\left.+\psi_{\theta, s}\left(\boldsymbol{K}, \boldsymbol{K}^{\prime \prime \prime}, t\right) \psi_{\theta, \boldsymbol{r}}\left(\boldsymbol{K}^{\prime}-\boldsymbol{K}^{\prime \prime}, \boldsymbol{K}^{\prime \prime}-\boldsymbol{K}^{\prime \prime \prime}, t\right)\right\} \mathrm{d} \boldsymbol{K}^{\prime \prime \prime}\right] \mathrm{d} \boldsymbol{K}^{\prime \prime}
\end{aligned}
$$

and

$$
\begin{aligned}
& A_{2}\left(\boldsymbol{K}, \boldsymbol{K}^{\prime}, t\right)=-2 K_{l} K_{m}^{\prime}\left[\psi_{\theta l}(\boldsymbol{K}, t) \psi_{\theta m}\left(\boldsymbol{K}^{\prime}, t\right)\right]- \\
& -\int K_{l}\left(K_{t}-K_{t}^{\prime \prime}\right)\left[\psi_{\theta, l}\left(\boldsymbol{K}^{\prime}, \boldsymbol{K}^{\prime \prime}, t\right) \psi_{\theta t}\left(\boldsymbol{K}-\boldsymbol{K}^{\prime \prime}, t\right)\right] \mathrm{d} \boldsymbol{K}^{\prime \prime}- \\
& \quad-\int K_{l} K_{n}^{\prime \prime}\left[\delta_{l r}-\frac{K_{l}^{\prime \prime} K_{r}^{\prime \prime}}{K^{\prime \prime 2}}\right]\left[\psi_{r n}\left(\boldsymbol{K}^{\prime \prime}, t\right) \psi_{\theta, \theta}\left(\boldsymbol{K}-\boldsymbol{K}^{\prime \prime}, \boldsymbol{K}^{\prime}, t\right)\right] \mathrm{d} \boldsymbol{K}^{\prime \prime}- \\
& -\int K_{m}^{\prime}\left(K_{s}^{\prime}-K_{s}^{\prime \prime}\right)\left[\psi_{\theta s}\left(\boldsymbol{K}^{\prime}-\boldsymbol{K}^{\prime \prime}, t\right) \psi_{\theta, m}\left(\boldsymbol{K}, \boldsymbol{K}^{\prime \prime}, t\right)\right] \mathrm{d} \boldsymbol{K}^{\prime \prime}- \\
& -\int K_{m}^{\prime} K_{s}^{\prime \prime}\left[\delta_{m r}-\frac{K_{m}^{\prime \prime} K_{r}^{\prime \prime}}{K^{\prime \prime 2}}\right]\left[\psi_{r s}\left(\boldsymbol{K}^{\prime \prime}, t\right) \psi_{\theta, \theta}\left(\boldsymbol{K}, \boldsymbol{K}^{\prime}-\boldsymbol{K}^{\prime \prime}, t\right)\right] \mathrm{d} \boldsymbol{K}^{\prime \prime}
\end{aligned}
$$

A similar procedure is followed to obtain the early-period decay equation for the spectrum $\psi_{\theta, j}\left(\boldsymbol{K}, \boldsymbol{K}^{\prime}, t\right)$ corresponding to the correlation $\overline{\theta u_{j}^{\prime}}\left(\boldsymbol{x}, \boldsymbol{x}^{\prime}, t\right)$ as

$$
\frac{\partial^{2}}{\partial t^{2}} \psi_{\theta, j}\left(\boldsymbol{K}, \boldsymbol{K}^{\prime}, t\right)=B_{1}\left(\boldsymbol{K}, \boldsymbol{K}^{\prime}, t\right)+B_{2}\left(\boldsymbol{K}, \boldsymbol{K}^{\prime}, t\right)
$$

where 


$$
\begin{aligned}
& B_{1}\left(K, K^{\prime}, t\right)=2 K_{i} K_{m}^{\prime}\left[\frac{K_{j}^{\prime} K_{q}^{\prime}}{K^{\prime 2}}-\delta_{j q}\right] \iint\left[\psi_{\theta, q}\left(K-K^{\prime \prime \prime}, K^{\prime}-K^{\prime \prime}, t\right) \cdot\right. \\
& \cdot \psi_{m, l}\left(\boldsymbol{K}^{\prime \prime}, \boldsymbol{K}^{\prime \prime \prime}, t\right)+\psi_{\theta, m}\left(\boldsymbol{K}-\boldsymbol{K}^{\prime \prime \prime}, \boldsymbol{K}^{\prime \prime}, t\right) \text {. } \\
& \left.\cdot \psi_{q, i}\left(\boldsymbol{K}^{\prime}-\boldsymbol{K}^{\prime \prime}, \boldsymbol{K}^{\prime \prime}, t\right)\right] \mathrm{d} \boldsymbol{K}^{\prime \prime} \mathrm{d} \boldsymbol{K}^{\prime \prime \prime}- \\
& -\int K_{l}\left(K_{t}-K_{t}^{\prime \prime}\right)\left[\int \left\{\psi_{\theta, j}\left(\boldsymbol{K}-\boldsymbol{K}^{\prime \prime}-\boldsymbol{K}^{\prime \prime \prime}, \boldsymbol{K}^{\prime}, t\right) \psi_{t, t}\left(\boldsymbol{K}^{\prime \prime}, \boldsymbol{K}^{\prime \prime \prime}, t\right)+\right.\right. \\
& \left.\left.+\psi_{\theta, l}\left(\boldsymbol{K}-\boldsymbol{K}^{\prime \prime}-\boldsymbol{K}^{\prime \prime \prime}, \boldsymbol{K}^{\prime \prime}, t\right) \psi_{j, t}\left(\boldsymbol{K}^{\prime}, \boldsymbol{K}^{\prime \prime \prime}, t\right)\right\} \mathrm{d} \boldsymbol{K}^{\prime \prime \prime}\right] \mathrm{d} \boldsymbol{K}^{\prime \prime}+ \\
& +\int\left(K _ { l } K _ { n } ^ { \prime \prime } [ \frac { K _ { l } ^ { \prime \prime } K _ { r } ^ { \prime \prime } } { K ^ { \prime \prime 2 } } - \delta _ { l r } ] \int \left\{\psi_{\theta, r}\left(K-K^{\prime \prime}, \boldsymbol{K}^{\prime \prime}-\boldsymbol{K}^{\prime \prime \prime}, t\right) \psi_{j, n} .\right.\right. \\
& \cdot\left(\boldsymbol{K}^{t}, \boldsymbol{K}^{\prime \prime t}, t\right)+ \\
& \left.\left.+\psi_{\theta, n}\left(\boldsymbol{K}-\boldsymbol{K}^{\prime \prime}, \boldsymbol{K}^{\prime \prime \prime}, t\right) \psi_{j, r}\left(\boldsymbol{K}^{\prime}, \boldsymbol{K}^{\prime \prime}-\boldsymbol{K}^{\prime \prime \prime}, t\right)\right\} \mathrm{d} \boldsymbol{K}^{\prime \prime}\right) \mathrm{d} \boldsymbol{K}^{\prime \prime}+ \\
& +\int\left(\left(K_{s}^{\prime}-K_{s}^{\prime \prime}\right)\left[\frac{\left(K_{q}^{\prime}-K_{q}^{\prime \prime}\right)\left(K_{r}^{\prime}-K_{r}^{\prime \prime}\right)}{\left(K^{\prime}-K^{\prime \prime}\right)^{2}}-\delta_{q r}\right] K_{m}^{\prime}\left[\partial_{j q}-\frac{K_{j}^{\prime} K_{q}^{\prime}}{K^{\prime 2}}\right]\right. \\
& \int\left\{\psi_{0, r}\left(\boldsymbol{K}, \boldsymbol{K}^{\prime}-\boldsymbol{K}^{\prime \prime}-\boldsymbol{K}^{\prime \prime \prime}, t\right) \psi_{m, s}\left(\boldsymbol{K}^{\prime \prime}, \boldsymbol{K}^{\prime \prime \prime}, t\right)+\right. \\
& \left.\left.+\psi_{0, s}\left(\boldsymbol{K}, \boldsymbol{K}^{\prime \prime \prime}, t\right) \psi_{r, m}\left(\boldsymbol{K}^{\prime}-\boldsymbol{K}^{\prime \prime}-\boldsymbol{K}^{\prime \prime \prime}, \boldsymbol{K}^{\prime \prime}, t\right)\right\} \mathrm{d} \boldsymbol{K}^{\prime \prime \prime}\right) \mathrm{d} \boldsymbol{K}^{\prime \prime}+ \\
& +\int\left(K _ { s } ^ { \prime \prime } [ \frac { K _ { m } ^ { \prime \prime } K _ { n } ^ { \prime \prime } } { K ^ { \prime \prime 2 } } - \delta _ { m n } ] K _ { m } ^ { \prime } [ \delta _ { j q } - \frac { K _ { j } ^ { \prime } K _ { q } ^ { \prime } } { K ^ { \prime 2 } } ] \int \left\{\psi_{\theta, s}\left(K, K^{\prime \prime \prime}, t\right) .\right.\right. \\
& \cdot \psi_{q, n}\left(\boldsymbol{K}^{\prime}-\boldsymbol{K}^{\prime \prime}, \boldsymbol{K}^{\prime \prime}-\boldsymbol{K}^{\prime \prime}, t\right)-\psi_{\theta, n}\left(\boldsymbol{K}, \boldsymbol{K}^{\prime \prime}-\boldsymbol{K}^{\prime \prime}, t\right) \cdot \\
& \left.\left.\psi_{q, s}\left(\boldsymbol{K}^{\prime}-\boldsymbol{K}^{\prime \prime}, \boldsymbol{K}^{\prime \prime}, t\right)\right\} \mathrm{d} \boldsymbol{K}^{\prime \prime \prime}\right) \mathrm{d} \boldsymbol{K}^{\prime \prime}
\end{aligned}
$$

and

$$
\begin{aligned}
& B_{2}\left(\boldsymbol{K}, \boldsymbol{K}^{\prime}, t\right)=2 K_{l} K_{m}^{\prime}\left[\frac{K_{j}^{\prime} K_{q}^{\prime}}{K^{\prime 2}}-\partial_{j q}\right] \psi_{\theta l}(\boldsymbol{K}, t) \psi_{q m}\left(\boldsymbol{K}^{\prime}, t\right)- \\
& \quad-\int K_{l}\left(K_{t}-K_{t}^{\prime \prime}\right)\left[\psi_{j, l}\left(\boldsymbol{K}^{\prime}, \boldsymbol{K}^{\prime \prime}, t\right) \psi_{\theta t}\left(\boldsymbol{K}-\boldsymbol{K}^{\prime \prime}, t\right)\right] \mathrm{d} \boldsymbol{K}^{\prime \prime}+ \\
& \quad+\int\left(K_{l} K_{n}^{\prime \prime}\left[\frac{K_{l}^{\prime \prime} K_{r}^{\prime \prime}}{K^{\prime \prime 2}}-\delta_{i \mathbf{r}}\right] \psi_{\theta, j}\left(\boldsymbol{K}-\boldsymbol{K}^{\prime \prime}, \boldsymbol{K}^{\prime}, t\right) \psi_{m n}\left(\boldsymbol{K}^{\prime \prime}, t\right)\right) \mathrm{d} \boldsymbol{K}^{\prime \prime}+
\end{aligned}
$$




$$
\begin{aligned}
& +\int\left(\left(K_{s}^{\prime}-K_{s}^{\prime \prime}\right)\left[\frac{\left(K_{q}^{\prime}-K_{q}^{\prime \prime}\right)\left(K_{r}^{\prime}-K_{r}^{\prime \prime}\right)}{\left(K^{\prime}-K^{\prime \prime}\right)^{2}}-\partial_{q r}\right] K_{m}^{\prime}\left[\delta_{j q}-\frac{K_{j}^{\prime} K_{q}^{\prime}}{K^{\prime 2}}\right]\right. \\
& \left.\cdot \psi_{\theta, m}\left(\boldsymbol{K}, \boldsymbol{K}^{\prime \prime}, t\right) \psi_{r s}\left(\boldsymbol{K}^{\prime}-\boldsymbol{K}^{\prime \prime}, t\right)\right) \mathrm{d} \boldsymbol{K}^{\prime \prime}+ \\
& +\int\left(K_{s}^{\prime \prime}\left[\frac{K_{m}^{\prime \prime} K_{n}^{\prime \prime}}{K^{\prime \prime}}-\delta_{m n}\right] K_{m}^{\prime}\left[\partial_{j q}-\frac{K_{j}^{\prime} K_{q}^{\prime}}{K^{\prime 2}}\right] \psi_{\theta, q}\left(\boldsymbol{K}, \boldsymbol{K}^{\prime}-\boldsymbol{K}^{\prime \prime}, t\right) \cdot\right. \\
& \left.\cdot \psi_{n s}\left(\boldsymbol{K}^{\prime \prime}, t\right)\right) \mathrm{d} \boldsymbol{K}^{\prime \prime}
\end{aligned}
$$

The equations (2.30) and (2.31) along with the equation derived by Ghosh [12] for the spectrum $\psi_{i, j}\left(\boldsymbol{K}, \boldsymbol{K}^{\prime}, t\right)$.

[Corresponding to the correlation $\overline{u_{i} u_{j}^{\prime}}\left(\boldsymbol{x}, \boldsymbol{x}^{\prime}, t\right)$ ]

$$
\frac{\partial^{2}}{\partial t^{2}} \psi_{i, j}\left(\boldsymbol{K}, \boldsymbol{K}^{\prime}, t\right)=I_{1}\left(\boldsymbol{K}, \boldsymbol{K}^{\prime}, t\right)+I_{2}\left(\boldsymbol{K}, \boldsymbol{K}^{\prime}, t\right)
$$

form a complete set of equations depicting the early-period decay process of a general type of turbulence.

\section{§ 3. Reduction of equations for the case of homogeneous isotropic turbulence}

\section{a) Case of homogeneous turbulence}

The equations obtained in the last section are simplified firstly for the case of homogeneous turbulence and subsequently for the case of homogeneous and isotropic turbulence. It is known that in homogeneous turbulence the correlation functions $\overline{u_{i} u_{j}^{\prime}}, \overline{\theta u_{j}^{\prime}}$ and $\overline{\theta \theta^{\prime}}$ formed of the physical entities pertaining to the points $P(x, t)$ and $P^{\prime}\left(x^{\prime}, t\right)$ are not separately dependent on $\boldsymbol{x}$ and $\boldsymbol{x}^{\prime}$ but only on the separation vector $\xi \equiv \boldsymbol{x}^{\prime}-\boldsymbol{x}$. The implicit dependence on $t$ is of course remaining unchanged. So, we may introduce three dimentional Dirac-delta function of the form $\delta\left(\boldsymbol{K}+\boldsymbol{K}^{\prime}\right)$ [cf. Ghosh [12]] for the reduction of (2.30) to (2.32) to the case of homogeneous turbulence. Before integration over the whole of $\boldsymbol{k}, \boldsymbol{k}^{\prime}$ spaces the functions of the types $\psi_{\theta, \theta}\left(\boldsymbol{K}, \boldsymbol{K}^{\prime}, t\right), \psi_{\theta, j}\left(\boldsymbol{K}, \boldsymbol{K}^{\prime}, t\right)$ and $\psi_{i, j}\left(\boldsymbol{K}, \boldsymbol{K}^{\prime}, t\right)$ etc. appearing in (2.30) to (2.32) are to be replaced respectively by

$$
\begin{aligned}
& \psi_{\theta, \theta}\left(\boldsymbol{K}, \boldsymbol{K}^{\prime}, t\right) \delta\left(\boldsymbol{K}+\boldsymbol{K}^{\prime}\right), \quad \psi_{\theta, j}\left(\boldsymbol{K}, \boldsymbol{K}^{\prime}, t\right) \delta\left(\boldsymbol{K}+\boldsymbol{K}^{\prime}\right) \\
& \text { and } \quad \psi_{i, j}\left(\boldsymbol{K}, \boldsymbol{K}^{\prime}, t\right) \delta\left(\boldsymbol{K}+\boldsymbol{K}^{\prime}\right) \quad \text { etc. }
\end{aligned}
$$


Taking this into consideration, we obtain after usual calculation the reduced versions of equations (2.30) and (2.31) in the case of homogeneous turbulence as

$$
\begin{aligned}
& \frac{\partial^{2}}{\partial t^{2}} \int \psi_{\theta, \theta}(\boldsymbol{K},-\boldsymbol{K}, t) \mathrm{d} \boldsymbol{K}= \\
& \quad=2 \iint K_{l}^{\mathrm{iv}} K_{m}^{\mathrm{iv}}\left\{\psi_{\theta, \theta}(\boldsymbol{K},-\boldsymbol{K}, t) \psi_{m, l}\left(-\boldsymbol{K}^{\prime}, \boldsymbol{K}^{\prime}, t\right)+\right. \\
& \left.\quad+\psi_{\theta, m}\left(\boldsymbol{K}^{\prime},-\boldsymbol{K}^{\prime}, t\right) \psi_{\theta, l}(-\boldsymbol{K}, \boldsymbol{K}, t)\right\} \mathrm{d} \boldsymbol{K} \mathrm{d} \boldsymbol{K}^{\prime}- \\
& \quad-\iint K_{l} K_{t}^{\mathrm{iv}}\left\{\psi_{\theta, \theta}(\boldsymbol{K},-\boldsymbol{K}, t) \psi_{l, t}\left(-\boldsymbol{K}^{\prime}, \boldsymbol{K}^{\prime}, t\right)+\right. \\
& \left.\quad+\psi_{\theta, l}\left(\boldsymbol{K}^{\prime},-\boldsymbol{K}^{\prime}, t\right) \psi_{\theta, t}(-\boldsymbol{K}, \boldsymbol{K}, t)\right\} \mathrm{d} \boldsymbol{K} \mathrm{d} \boldsymbol{K}^{\prime}- \\
& \quad-\iint K_{l} \boldsymbol{K}_{n}^{\mathrm{iv}}\left[\delta_{l r}-\frac{K_{l}^{\mathrm{iv}} K_{r}^{\mathrm{iv}}}{K^{\mathrm{iv}}}\right]\left\{\psi_{\theta, r}\left(-\boldsymbol{K}^{\prime}, \boldsymbol{K}^{\prime}, t\right) \psi_{\theta, n}(-\boldsymbol{K}, \boldsymbol{K}, t)+\right. \\
& \left.\quad+\psi_{\theta, n}\left(-\boldsymbol{K}^{\prime}, \boldsymbol{K}^{\prime}, t\right) \psi_{\theta, r}(-\boldsymbol{K}, \boldsymbol{K}, t)\right\} \mathrm{d} \boldsymbol{K} \mathrm{d} \boldsymbol{K}^{\prime}- \\
& \quad-\iint K_{m} K_{s}^{\mathrm{iv}}\left\{\psi_{\theta, \theta}(\boldsymbol{K},-\boldsymbol{K}, t) \psi_{m, s}\left(\boldsymbol{K}^{\prime},-\boldsymbol{K}^{\prime}, t\right)+\right. \\
& \left.\quad+\psi_{\theta, s}(\boldsymbol{K},-\boldsymbol{K}, t) \psi_{\theta, m}\left(-\boldsymbol{K}^{\prime}, \boldsymbol{K}^{\prime}, t\right)\right\} \mathrm{d} \boldsymbol{K} \mathrm{d} \boldsymbol{K}^{\prime}- \\
& \quad-\iint K_{m} K_{s}^{\mathrm{iv}}\left[\delta_{m r}-\frac{K_{m}^{\mathrm{iv}} K_{r}^{\mathrm{iv}}}{\boldsymbol{K}^{\mathrm{iv}}}\right]\left\{\psi_{\theta, s}(-\boldsymbol{K}, \boldsymbol{K}, t) \psi_{\theta, r}\left(-\boldsymbol{K}^{\prime}, \boldsymbol{K}^{\prime}, t\right)+\right. \\
& \left.\quad+\psi_{\theta, r}(-\boldsymbol{K}, \boldsymbol{K}, t) \psi_{\theta, s}\left(-\boldsymbol{K}^{\prime}, \boldsymbol{K}^{\prime}, t\right)\right\} \mathrm{d} \boldsymbol{K} \mathrm{d} \boldsymbol{K}^{\prime}
\end{aligned}
$$

and

$$
\begin{aligned}
& \frac{\partial^{2}}{\partial t^{2}} \int \psi_{\theta, j}(\boldsymbol{K},-\boldsymbol{K}, t) \mathrm{d} \boldsymbol{K}= \\
& \quad=-\iint K_{l} K_{t}^{\mathrm{iv}}\left\{\psi_{\theta, j}(\boldsymbol{K},-\boldsymbol{K}, t) \psi_{l, t}\left(-\boldsymbol{K}^{\prime}, \boldsymbol{K}^{\prime}, t\right)+\right. \\
& \left.\quad+\psi_{\theta, l}\left(\boldsymbol{K}^{\prime},-\boldsymbol{K}^{\prime}, t\right) \psi_{j, t}(-\boldsymbol{K}, \boldsymbol{K}, t)\right\} \mathrm{d} \boldsymbol{K} \mathrm{d} \boldsymbol{K}^{\prime}- \\
& \quad-\iint 2 K_{l}^{\mathrm{iv}} K_{m}^{\mathrm{iv}}\left[\frac{K_{j}^{\mathrm{iv}} K_{q}^{\mathrm{iv}}}{K^{\mathrm{iv} z}}-\delta_{j q}\right]\left\{\psi_{\theta, q}(\boldsymbol{K},-\boldsymbol{K}, t) \psi_{m, l}\left(-\boldsymbol{K}^{\prime}, \boldsymbol{K}^{\prime}, t\right)+\right. \\
& \left.\quad+\psi_{\theta, m}\left(\boldsymbol{K}^{\prime},-\boldsymbol{K}^{\prime}, t\right) \psi_{q, l}(-\boldsymbol{K}, \boldsymbol{K}, t)\right\} \mathrm{d} \boldsymbol{K} \mathrm{d} \boldsymbol{K}^{\prime}+
\end{aligned}
$$




$$
\begin{aligned}
& +\iint K_{l} K_{n}^{\mathrm{iv}}\left[\frac{K_{l}^{\mathrm{iv}} K_{r}^{\mathrm{iv}}}{K^{\mathrm{iv}}}-\delta_{l r}\right]\left\{\psi_{\theta, r}\left(-\boldsymbol{K}^{\prime}, \boldsymbol{K}^{\prime}, t\right) \psi_{j, n}(-\boldsymbol{K}, \boldsymbol{K}, t)+\right. \\
& \left.+\psi_{\theta, n}\left(-\boldsymbol{K}^{\prime}, \boldsymbol{K}^{\prime}, t\right) \psi_{j, r}(-\boldsymbol{K}, \boldsymbol{K}, t)\right\} \mathrm{d} \boldsymbol{K} \mathrm{d} \boldsymbol{K}^{\prime}+ \\
& +\iint K_{s}^{\mathrm{iv}}\left[\frac{K_{q}^{\mathrm{iv}} K_{r}^{\mathrm{iv}}}{K^{\mathrm{iv}}}-\delta_{q r}\right] K_{m}\left[\delta_{j q}-\frac{K_{j} K_{q}}{K^{2}}\right]\left\{\psi_{\theta, r}(\boldsymbol{K},-\boldsymbol{K}, t) \cdot\right. \\
& \left.+\psi_{m, s}\left(\boldsymbol{K}^{\prime},-\boldsymbol{K}^{\prime}, t\right)+\psi_{\theta, s}(\boldsymbol{K},-\boldsymbol{K}, t) \psi_{\boldsymbol{r}, m}\left(-\boldsymbol{K}^{\prime}, \boldsymbol{K}^{\prime}, t\right)\right\} \mathrm{d} \boldsymbol{K} \mathrm{d} \boldsymbol{K}^{\prime}+ \\
& +\iint K_{s}^{\mathrm{iv}}\left[\frac{K_{m}^{\mathrm{iv}} K_{n}^{\mathrm{iv}}}{\boldsymbol{K}^{\mathrm{iv}}{ }^{\mathrm{i}}}-\delta_{m n}\right] K_{m}\left[\delta_{j q}-\frac{K_{j} K_{q}}{\boldsymbol{K}^{2}}\right]\left\{\psi_{\theta, s}(-\boldsymbol{K}, \boldsymbol{K}, t) \cdot\right. \\
& \left.+\psi_{q, n}\left(-\boldsymbol{K}^{\prime}, \boldsymbol{K}^{\prime}, t\right)+\psi_{\theta, n}(-\boldsymbol{K}, \boldsymbol{K}, t) \psi_{q, s}\left(-\boldsymbol{K}^{\prime}, \boldsymbol{K}^{\prime}, t\right)\right\} \mathrm{d} \boldsymbol{K} \mathrm{d} \boldsymbol{K}^{\prime}
\end{aligned}
$$

where $\boldsymbol{K}^{\mathrm{ir}}=\boldsymbol{K}+\boldsymbol{K}^{\prime}$.

The reduced form of (2.32) in the case of homogeneous turbulence is

$$
\begin{aligned}
& \frac{\partial^{2}}{\partial t^{2}} \int \psi_{i, j}(\boldsymbol{K},-\boldsymbol{K}, t) \mathrm{d} \boldsymbol{K}= \\
& =2 \iint K_{l}^{\mathrm{iv}}\left[\delta_{i s}-\frac{K_{i}^{\mathrm{iv}} K_{s}^{\mathrm{iv}}}{K^{\mathrm{iv}}}\right] K_{m}^{\mathrm{iv}}\left[\delta_{j q}-\frac{K_{j}^{\mathrm{iv}} K_{q}^{\mathrm{iv}}}{K^{\mathrm{iv}{ }^{2}}}\right]\left\{\psi_{s, q}(K,-K, t) \cdot\right. \\
& \left.\cdot \psi_{m, l}\left(-\boldsymbol{K}^{\prime}, \boldsymbol{K}^{\prime}, t\right)+\psi_{s, m}(\boldsymbol{K},-\boldsymbol{K}, t) \psi_{q, l}\left(-\boldsymbol{K}^{\prime}, \boldsymbol{K}^{\prime}, t\right)\right\} \mathrm{d} \boldsymbol{K} \mathrm{d} \boldsymbol{K}^{\prime}+ \\
& +\iint K_{t}^{\mathrm{iv}}\left[\frac{K_{s}^{\mathrm{iv}} K_{q}^{\mathrm{iv}}}{K^{\mathrm{iv}^{2}}}-\delta_{s q}\right] K_{l}\left[\delta_{i s}-\frac{K_{i} K_{s}}{K^{2}}\right]\left\{\psi_{q, j}(\boldsymbol{K},-\boldsymbol{K}, t) .\right. \\
& \left.\cdot \psi_{l, t}\left(-\boldsymbol{K}^{\prime}, \boldsymbol{K}^{\prime}, t\right)+\psi_{q, l}\left(\boldsymbol{K}^{\prime},-\boldsymbol{K}^{\prime}, t\right) \psi_{j, t}(-\boldsymbol{K}, \boldsymbol{K}, t)\right\} \mathrm{d} \boldsymbol{K} \mathrm{d} \boldsymbol{K}^{\prime}+ \\
& +\iint K_{n}^{\mathrm{iv}}\left[\frac{K_{r}^{\mathrm{iv}} K_{l}^{\mathrm{iv}}}{K^{\mathrm{iv} \mathrm{v}^{2}}}-\delta_{r l}\right] K_{l}\left[\delta_{i s}-\frac{K_{i} K_{s}}{K^{2}}\right]\left\{\psi_{s, r}\left(-K^{\prime}, K^{\prime}, t\right) .\right. \\
& \left.\cdot \psi_{j, n}(-\boldsymbol{K}, \boldsymbol{K}, t)+\psi_{s, n}\left(-\boldsymbol{K}^{\prime}, \boldsymbol{K}^{\prime}, t\right) \psi_{j, r}(-\boldsymbol{K}, \boldsymbol{K}, t)\right\} \mathrm{d} \boldsymbol{K} \mathrm{d} \boldsymbol{K}^{\prime}+ \\
& +\iint K_{s}^{\mathrm{iv}}\left[\frac{K_{q}^{\mathrm{iv}} K_{\mathrm{t}}^{\mathrm{iv}}}{K^{\mathrm{iv}^{2}}}-\delta_{q t}\right] K_{m}\left[\delta_{j q}-\frac{K_{j} K_{q}}{K^{2}}\right]\left\{\psi_{i, t}(\boldsymbol{K},-\boldsymbol{K}, t) .\right. \\
& \left.\cdot \psi_{m, s}\left(\boldsymbol{K}^{\prime},-\boldsymbol{K}^{\prime}, t\right)+\psi_{i, \mathrm{~s}}(\boldsymbol{K},-\boldsymbol{K}, t) \psi_{t, m}\left(-\boldsymbol{K}^{\prime}, \boldsymbol{K}^{\prime}, t\right)\right\} \mathrm{d} \boldsymbol{K} \mathrm{d} \boldsymbol{K}^{\prime}+ \\
& +\iint K_{s}^{\mathrm{iv}}\left[\frac{K_{r}^{\mathrm{iv}} K_{m}^{\mathrm{iv}}}{K^{\mathrm{iv} 2}}-\delta_{r m}\right] K_{m}\left[\delta_{j q}-\frac{K_{j} K_{q}}{K^{2}}\right]\left\{\psi_{i, r}(K,-K, t) .\right. \\
& \left.\cdot \psi_{q, s}\left(\boldsymbol{K}^{\prime},-\boldsymbol{K}^{\prime}, t\right)+\psi_{i, s}(\boldsymbol{K},-\boldsymbol{K}, t) \psi_{q, r}\left(\boldsymbol{K}^{\prime},-\boldsymbol{K}^{\prime}, t\right)\right\} \mathrm{d} \boldsymbol{K} \mathrm{d} \boldsymbol{K}^{\prime}
\end{aligned}
$$

[cf. Ghosh [12]]. 
It is to be noticed that $A_{2}\left(K, K^{\prime}, t\right), B_{2}\left(K, K^{\prime}, t\right)$ and $I_{2}\left(K, K^{\prime}, t\right)$ appearing respectively in $(2.30),(2.31)$ and $(2.32)$ have got no contributions in case of homogeneous turbulence since they disappear when requisite integrals are computed.

b) Case of homogeneous and isotropic turbulence

We know that the turbulence is statistically homogeneous and isotropic if the turbulence field characteristics are independent of choice of origin of the frame of reference and all sorts of proper and improper rotations. In such case, the correlation tensors of the form $\overline{\theta u_{j}^{\prime}}$ can be found to vanish identically. So we need simplify equations (2.30) and (2.32) for the case of homogeneous and isotropic turbulence. To obtain results in a neat form, we nultiply (2.30) by $k^{2}$ and follow the same procedure as before and obtain

$$
\begin{aligned}
& \frac{\partial^{2}}{\partial t^{2}} \int K^{2} \psi_{\theta, \theta}(\boldsymbol{K},-\boldsymbol{K}, t) \mathrm{d} \boldsymbol{K}= \\
& \quad=\iint 2 K^{\mathrm{iv} 2} K_{l}^{\mathrm{iv}} K_{m}^{\mathrm{iv}} \psi_{\theta, \theta}(\boldsymbol{K},-\boldsymbol{K}, t) \psi_{m, l}\left(-\boldsymbol{K}^{\prime}, \boldsymbol{K}^{\prime}, t\right) \mathrm{d} \boldsymbol{K} \mathrm{d} \boldsymbol{K}^{\prime}- \\
& \quad-\iint K^{2} K_{l} K_{t}^{\mathrm{iv}} \psi_{\theta, \theta}(\boldsymbol{K},-\boldsymbol{K}, t) \psi_{l, t}\left(-\boldsymbol{K}^{\prime}, \boldsymbol{K}^{\prime}, t\right) \mathrm{d} \boldsymbol{K} \mathrm{d} \boldsymbol{K}^{\prime}- \\
& \quad-\iint K^{2} K_{s}^{\mathrm{iv}} K_{m} \psi_{\theta, \theta}(\boldsymbol{K},-\boldsymbol{K}, t) \psi_{m, s}\left(\boldsymbol{K}^{\prime},-\boldsymbol{K}^{\prime}, t\right) \mathrm{d} \boldsymbol{K} \mathrm{d} \boldsymbol{K}^{\prime}
\end{aligned}
$$

We now introduce the usual isotropic forms for the spectrum tensors $\psi_{\theta, \theta}(K,-K, t)$ and $\psi_{m, l}\left(-\boldsymbol{K}^{\prime}, \boldsymbol{K}^{\prime}, t\right)$ as

$$
\psi_{\theta, \theta}(K,-K, t)=\frac{1}{4 \pi K^{2}} E_{\theta \theta}(K, t)
$$

and

$$
\psi_{m, l}\left(-\boldsymbol{K}^{\prime}, \boldsymbol{K}^{\prime}, t\right)=\frac{F\left(K^{\prime}, t\right)}{4 \pi K^{\prime 2}}\left\{\delta_{m l}-\frac{K_{m}^{\prime} K_{l}^{\prime}}{K^{\prime 2}}\right\}
$$

where $E_{\theta \theta}(K, t)$ and $F\left(K^{\prime}, t\right)$ are respectively thermal energy spectrum and kinetic energy spectrum. Using (3.5), (3.6) and expressions similar to (3.6) for the second rank spectrum tensors appearing in (3.4), we deduce from (3.4) an equation of the form, 


$$
\begin{aligned}
& \frac{\partial^{2}}{\partial t^{2}} \int_{0}^{\infty} K^{2} E_{\theta \theta}(K, t) \mathrm{d} K= \\
& =\int_{0}^{\infty} \int_{0}^{\infty} \int_{-1}^{1}-\frac{Q}{4 K^{\prime 2}}\left\{K^{\mathrm{iv} 2}-K^{2}\right\} E_{\theta \theta}(K, t) F\left(K^{\prime}, t\right) \mathrm{d} K \mathrm{~d} K^{\prime} \mathrm{d} \mu
\end{aligned}
$$

where the space integral $\mathrm{d} K$ has been replaced by the line integral $4 \pi K^{2} \mathrm{~d} K$ and the multiple integral $\mathrm{d} \boldsymbol{K} \mathrm{d} \boldsymbol{K}^{\prime}$ has been replaced by $4 \pi K^{2} \mathrm{~d} K$. $\cdot 2 \pi K^{\prime 2} \mathrm{~d} K^{\prime} \mathrm{d} \mu, \mu$ being the cosine of the angle between $\boldsymbol{k} \& \boldsymbol{k}^{\prime}$ and $Q$ is a symmetric form given by

$$
Q=K^{4}+K^{\prime 4}+K^{\mathrm{iv} 4}-2 K^{2} K^{\prime 2}-2 K^{\prime 2} K^{\mathrm{iv} 2}-2 K^{\mathrm{iv}^{2}} K^{2}
$$

After integrations, (3.7) is reducible to the simple form

$$
\frac{\partial^{2}}{\partial t^{2}} \int_{0}^{\infty} K^{2} E_{\theta \theta}(K, t) \mathrm{d} K=\frac{4}{3} \int_{0}^{\infty} K^{2} E_{\theta \theta}(K, t) \mathrm{d} K \int_{0}^{\infty} K^{2} F(K, t) \mathrm{d} K
$$

which depicts the early-period decay phenomenon for the thermal energy $E_{\theta \theta}(K, t)$ in isotropic turbulence field.

Equation (3.9) is the same as the standard decay equation derived for a scalar field in Statistical Fluid Mechanics, Vol. 2 (P 288, equation 19.61) by A. S. Monin \& A. M. Yaglom [13].

In the similar manner the early-period decay equation for the kinetic energy spectrum is obtained as

$$
\frac{\partial^{2}}{\partial t^{2}} \int_{0}^{\infty} 2 K^{2} F(K, t) \mathrm{d} K=\frac{4}{3}\left[\int_{0}^{\infty} K^{2} F(K, t) \mathrm{d} K\right]^{2}
$$

[cf. Reid \& Proudman [14], Tatsumi [15], Ghosh [12]].

\section{Acknowledgements}

The author is grateful to Prof. K. M. Ghosh for suggesting the problem and for constant encouragement throughout the progress of the work. He also thanks Professor Ambarish Ghosh and Prof. dr. ir. P. J. Zandbergen for stimulating discussions on this problem.

Received 13 September 1976 


\section{REFERENCES}

[1] Obuкhoff, A. M., Izv. Akad. Nauk. SSSR, Ser. Geofiz 13 (1949) 58.

[2] Corrsin, S., J. Appl. phys. 22 (1951) 469.

[3] O'Brien, E. E. and G. C. Francis, J. Fluid. Mech. 13 (1962) 369.

[4] Millionshtchiкov, M., Dokl. Akad. Nauk. SSSR 32 (1941a) 611.

[5] Millionshtchikov, M., Izv. Akad. Nauk. SSSR Series Geogr. Geophys. 5 (1941b) 433.

[6] Chandrasekhar, S., Proc. Roy. Soc. A 229 (1955) 1.

[7] J JIN, P. C., Proc. Nat. Instt. of Sci. of India A28 (1962) 401.

[8] Codreanu, S., Studia, Universitaties Babes-Bolyai, Series physica, Fasciculus 2 (1973) 69.

[9] MalfLiet, W. P. M., physica 45 (1969) 257.

[10] Kistler, A. L., V. O'Brien, and S. Corrsin, Natl. Advisory Comm. Aeronaut Research Mem. No. RM 54 (1954) D 19.

[11] PancheV, S., P.M.M. 33 (1969) 1091.

[12] Gноsн, K. M., Indian Journal of Pure and Appl. Math. 3 (1972) 157.

[13] Monin, A. S. and Yaglom, A. M., Statistical Fluid Mechanics 2, M.I.T. Press, 1975, p. 288.

[14] Reid, W. H. and I. Proudman, Phil. Trans. Roy. Soc. A247 (1954) 163.

[15] Tatsumi, T., Proc. Roy. Soc. A239 (1957) 16. 